\title{
Event-Level Substance Use and Unprotected Sex among Young Adults: A Case-Crossover Analysis
}

Muchimba ${ }^{1 *}$, Burton $\mathrm{M}^{2}$, Haberstick $\mathrm{BC}^{2}$, Corley $\mathrm{RP}^{2}$, Hopfer $\mathrm{C}^{3}$ and McQueen $\mathrm{M}^{2,4}$

${ }^{1}$ Department of Health Sciences, Saginaw Valley State University, University City, USA

${ }^{2}$ Institutes for Behavioral Genetics, University of Colorado Boulder, USA

${ }^{3}$ Department of Psychiatry, University of Colorado Denver, USA

${ }^{4}$ Department of Integrative Physiology, University of Colorado Boulder, USA

*Corresponding author: Muchimba M, Department of Health Sciences, Department of Health Sciences, Saginaw Valley State University, University City, USA

Received: J anuary 11, 2017; Accepted: February 09, 2017; Published: February 10, 2017

\begin{abstract}
Background: It has been theorized that the use of alcohol and other drugs during a sexual encounter increases the likelihood of unprotected sex. Few studies have examined the relationship between substance use and unprotected sex in clinical samples. This study investigated event-level substance use (alcohol and other drugs) and unprotected sex in community and clinical samples.
\end{abstract}

Methods: A sample of 2775 community and clinical participants aged 19 to 30 years was surveyed between 2008 and 2011. To assess event-level substance use and unprotected sex, we conducted a case-crossover analysis using conditional logistic regression.

Results: Results revealed no association between substance use and unprotected sex among male and female clinical subjects, and among female community subjects. Among community males, there was a negative association (OR, 0.69; 95\% Cl, 0.48-0.99).

Conclusion: STI/HIV preventive programs should not focus only on the consequences of engaging in sex while under the influence of alcohol and other drugs. To be more effective, they need to consider other factors which influence the relationship between substance use and unprotected sex, including type and amount of substances, personality, context of sexual encounter and type of relationship.

Keywords: Case-crossover; Event-level; Alcohol; Substance use; Unprotected sex

\section{Introduction}

Sexually Transmitted Infections (STIs) remain a public health concern in the United States, with nearly 20 million new Sexually Transmitted Infections (STIs) occurring every year [1]. Untreated STIs can lead to reproductive health complications, such as pelvic inflammatory disease, chronic pelvic pain, and infertility among women, and epididymitis and urethritis among men [2]. Human papillomavirus - the most common STI - can cause genital warts, cervical, and other cancers. STIs can also increase a person's risk for acquiring and transmitting HIV infection [3].

Correct and consistent use of condom remains one of the most effective methods to protect against STIs, including HIV, and a large body of research has focused on factors are that are associated with non-use of condoms. One factor that has been studied extensively is the use of Alcohol and Other Drugs (AOD). AOD are thought to interfere with judgment and decision-making when consumed, and it is believed that their use during sexual activity increases the likelihood that risky sexual behaviors, such unprotected sex, will occur [4].

Correlational studies have shown that the use of AOD is associated with non-use of condoms. For example, among men who have sex with men, the use of inhalants was associated with failure to use condoms during receptive anal sex [5]; and the use of alcohol, inhalants and non-injection drug use before or during sex were associated with significantly more unprotected anal sex [6]. Similar findings have been reported among young people. Per a Kaiser Family Foundation survey among 998 teens and young adults, 22\% said they had unprotected sex because they were drinking or using drugs [7].

Most of the research on the relationship between substance use and condom non-use is correlational, and is therefore limited in that the temporal relationship between the two behaviors is not examined. Thus, it cannot be determined whether substance use occurred shortly before unprotected sex, a condition that is necessary for establishing substance use as a risk factor. Event-level studies represent an improvement over correlational designs in that it temporally pairs use of AOD with condom non-use during a specific sexual encounter. Thus, these studies are better able to demonstrate whether the use of AOD preceded unprotected sex, thereby strengthening the causal interpretation [8]. Event-level studies do not establish causality; however, temporally pairing the two behaviors provides greater insight into whether substance use before sex increases the likelihood that unprotected sex will occur. This allows for a more indepth examination of the hypothesis that being under the influence of substances during sexual activity is associated with increased probability of sexual risk behavior.

Some event-level studies have found an association between the use of AOD and condom non-use while others have not. Findings from studies among general population participants have shown that 
participants were no more or less likely to use condoms during events involving use of AOD than during those without it [9]. However, positive associations have been found in some populations. Eventlevel use of AOD has been shown to predict unprotected sex among STI patients [10], and adolescents involved with the juvenile justice system [11]. Considering these mixed results, there is need for further exploration of the association of substance use with condom use at the event level.

Because of differences that exist between males and females regarding substance use and condom use, gender is an important variable to consider in research on AOD and condom use. Men are more likely than women to use almost all types of illicit drugs [12]. Also, the literature suggests that women are less likely to use condoms than men [13]. Barriers to condom use may also differ by gender. There is evidence that men are more likely than women to be concerned that condoms would interfere with sexual pleasure [14]. However, despite these gender differences, event-level studies that have analyzed data by gender are limited.

Even though substance use disorders increase the likelihood of HIV infection and of engaging risky sexual behaviors such as non-use of condoms and multiple sexual partners [15], few event-level studies have included clinical samples. Injection drug users still accounts for a substantial percentage of HIV infections. They comprise an estimated $2.6 \%$ of the U.S. population yet they account for $22 \%$ of all persons living with HIV infection [16]. In addition, the literature shows a link between STIs and the use of drugs such as cocaine [17]. This highlights the importance of examining the event-level relationship between substance use and condom use in clinical populations.

The purpose of the current investigation was to examine the relationship between substance use and unprotected sex using eventlevel data. This study will contribute to the research by examining the relationship by gender and by including a clinical sample.

\section{Methods}

\section{Sample and materials}

Participants included community and clinical samples, which were part of the Center for Antisocial Drug Dependence (CADD), an ongoing, multi-component, collaborative study at the University of Colorado. Community participants who were drawn from the twin, adoption, and family study components of the CADD. Twin participants were recruited from the Colorado Twin Registry [18], which consists of a community-based sample of twin families residing in Colorado. Non-twin participants were drawn from two community-based family samples: the Colorado Adoption Project [19], an ongoing, longitudinal study of the genetic and environmental influences on behavioral, cognitive, and emotional development; and the Colorado Adolescent Substance Abuse family study [20], a research of the familial transmission of substance abuse and associated psychopathology, which recruits adolescents in treatment and matched community controls. The present study comprised 2775 community and clinical participants, aged 19 to 30 years, who completed assessments between September 2008 and March 2011.

Participants were interviewed using the Modified Risk Behavior Questionnaire (M-RBQ), an instrument developed for the CADD, which includes a series of questions on risky sexual behaviors and substance-related STI/HIV behaviors [21]. All participants provided written informed consent prior to the interviews. This study was approved by the University of Colorado Institutional Review Board.

\section{Study variables}

Information on whether AOD were used during a sexual encounter was obtained from two questions. For the first question, participants were asked, "Recall the last time you had unprotected sex and did not use a condom for protection. Were you using alcohol or drugs at the time?" Answer options for this question were $0=\mathrm{No}$, $1=$ Yes, $3=$ I always use condoms so I can't answer this question yes or no. The second question was "Recall the last time you had unprotected sex and did use a condom for protection. Were you using alcohol or drugs at the time?" Answer options for this question were $0=$ No, $1=$ Yes, $3=I$ never use condoms so I can't answer this question yes or no. We also obtained information on age, gender, ethnicity and marital status.

\section{Statistical analysis}

Event-level associations between substance use and unprotected sex were examined by utilizing a case-crossover analysis. This approach allows each participant to serve as his or her own control, thereby eliminating confounding personal factors that are not time-varying. To be included in the analysis, participants had to report at least one unprotected and one protected sexual encounter. Conditional logistic regression models were fitted to estimate odds ratios (ORs) - with their corresponding 95\% confidence intervals (CIs) - of the likelihood of unprotected sex when substances were used. This was achieved by comparing participants' condom use when substances were used and their condom use when substances were not used. To observe whether the relationship between substance use and unprotected sex differed between community and clinical participants, and between males and females, we ran the analyses separately for community and clinical participants, and by gender in the two groups. Participants who reported always using condoms or never using condoms were left out of the analysis.

Statistical analysis was performed using R Statistical Software (version 2.15.1; Foundation for Statistical Computing, Vienna, Austria) [22].

\section{Results}

\section{Descriptive characteristics}

In the entire sample, 2250 (91.9\%) were community participants while 225(8.1) were clinical participants. Female's constituted 51.0\% of the overall sample (Table 1 ). In the community sample, $52.8 \%$ were female and over two-thirds (69.9\%) were aged 22 to 27 years (mean age $=25.0 ; \mathrm{SD}=2.6)$. Community participants were largely white $(86.6 \%)$ and over a third (69.7\%) had never been married. Most of the participants (93.3\%) were not using drugs at the time of the survey.

Among clinical participants, $32.6 \%$ were female and the majority $(77.2 \%)$ were aged 25 to 30 years (mean age $=26.3$; $\mathrm{SD}=2.7$ ). Like the community sample, most of the clinical participants were white (60.1\%). There were, however, notably higher proportions of Native Americans and African Americans in the clinical group than in the community group. Most (62.1\%) of the clinical participants had never married. Like community participants, most of the clinical participants (85.1\%) were currently not using drugs. 
Table 1: Sociodemographic characteristics of the study sample.

\begin{tabular}{|c|c|c|}
\hline \multirow{2}{*}{ Characteristic } & \multicolumn{2}{|c|}{ n (\%) } \\
\hline & Community subjects & Clinical subjects \\
\hline \multicolumn{3}{|l|}{ Gender } \\
\hline Male & $1205(47.25)$ & $154(68.44)$ \\
\hline Female & $1345(52.75)$ & $71(31.56)$ \\
\hline \multicolumn{3}{|l|}{ Age } \\
\hline $19-21$ & $280(10.98)$ & $19(8.44)$ \\
\hline $22-24$ & $818(32.03)$ & $33(14.67)$ \\
\hline $25-27$ & 967 (37.92) & $88(39.11)$ \\
\hline $28-30$ & $490(19.21)$ & $88(39.11)$ \\
\hline Mean & 24.98 & 26.33 \\
\hline SD & 2.57 & 2.72 \\
\hline \multicolumn{3}{|l|}{ Ethnicity } \\
\hline American Indian, Native Alaskan & $29(1.14)$ & $11(4.89)$ \\
\hline Asian & $29(1.14)$ & $0(0.00)$ \\
\hline Black & $35(1.37)$ & $17(7.56)$ \\
\hline White & $2211(86.71)$ & $137(60.90)$ \\
\hline Hispanic & $206(8.08)$ & $56(24.89)$ \\
\hline Unknown, not specified & $42(1.65)$ & $7(3.11)$ \\
\hline \multicolumn{3}{|l|}{ Marital status } \\
\hline Never married & 1777 (69.69) & 139 (61.78) \\
\hline Married/cohabitating & $684(26.82)$ & $58(25.78)$ \\
\hline Separated/divorced & 85 (3.33) & $28(12.45)$ \\
\hline Widowed & $4(0.16)$ & $0(0.00)$ \\
\hline
\end{tabular}

\section{Sexual behavior}

Of the 2775 participants, $1240(44.6 \%)$ reported always using condoms or never using condoms. Because this analysis required both case (unprotected) and control (protected) events, these participants were excluded from the analysis. Therefore, the subset of 1535 participants (55.3\%) who reported both unprotected and control protected sexual encounters constituted the sample for this analysis. Those in the final analysis subset were significantly more likely to be male, white, and married or living together.

\section{Associations between event-level substance use and unprotected sex}

Conditional logistic regression results of the associations between substance use during a sexual encounter and unprotected sex are depicted in Table 2. In the combined sample, substance use during a sexual encounter did not increase the likelihood of unprotected sex. Among female community participants, a positive association was revealed, however, these results failed to reach statistical significance (OR, 1.24; 95\% CI, 0.87-1.77). Results among community males indicated a negative association between substance use and unprotected sex -- not using a condom was significantly less likely to occur when substances were used compared to when they were not used (OR, 0.69; 95\% CI, 0.48-0.99). For clinical participants, results showed associations that were negative but not statistically significant among both males (OR, 0.67; 95\% CI, 0.27-1.63) and females (OR, 0.75 ; 95\% CI, 0.17-3.35).
Table 2: Conditional logistic regression results of event-level substance use and unprotected sex.

\begin{tabular}{|c|c|}
\hline Sample & OR (95\% Cl) \\
\hline All subjects & $0.90(0.71-1.15)$ \\
\hline Community & $0.93(0.72-1.19)$ \\
\hline Female community subjects & $1.24(0.87-1.77)$ \\
\hline Male community subjects & $0.69(0.48-0.99)$ \\
\hline Clinical & $0.69(0.32-1.48)$ \\
\hline Female clinical subjects & $0.75(0.17-3.35)$ \\
\hline Male clinical subjects & $0.67(0.27-1.63)$ \\
\hline
\end{tabular}

\section{Discussion}

This study examined the event-level association between the uses of AOD non-use of condoms. The theory that substance use prior to engaging in sexual intercourse is associated with increased odds of unprotected sex was not supported by the data among both community and clinical participants in the present study. This lends support to previous research that has observed no association between substance use and condom non-use [9].

It would be expected that use of AOD during sexual events would increase the likelihood of unprotected sex. However, evidence has largely not been convincing to date. There could be several explanations for the null findings in our study. The sample was mixed in terms of age, which ranged from 19 to 30 . Among both community and clinical samples - particularly in the clinical sample - most of the participants were older than 24 years. It is probable that older participants, who comprised the larger part of the overall sample, were in married or in steady relationships and were therefore less likely to use condoms. Another factor that could account for the findings is the relationship status of the participants. Even though most of the participants had reported never having been married, the probability that some of them may have been in steady relationships cannot be ruled out. There is evidence that condoms are used less often in serious relationships than they are in casual ones. For example, in one study, inconsistent or no condom use occurred in $13.9 \%$ of 2387 steady partnerships and in $33.5 \%$ of 4014 casual partnerships [23]. It is also plausible that targeted STD and HIV prevention efforts that promote the value and effectiveness of condoms have been effective [24]. These educational messages may have been successful to the extent that some individuals have learned to have protected sex consistently, whether they are under the influence of substances or not $[8,25]$. Or perhaps the alcohol myopia idea holds where some outcomes are concerned but not others. As Fortenberry et al. suggest [25], although substance use may promote risky behavior in settings such as in the operation of a motor vehicle, this may not be the case with behaviors such as condom use.

Of course, null findings do not discredit the view that substance use is associated with risky behavior. After all, research does show a relationship between use of AOD and unprotected sex in some populations at the event level. However, the mechanisms of the relationship between the two behaviors remain unclear. The relationship could be explained by underlying personality factors such as sensation seeking and impulsivity, which have been associated with both substance use [26] and sexual risk taking [27]. Further, despite 
the ambiguity of the substance use-unprotected sex relationship, the use of AOD could serve as a marker for a larger lifestyle associated with risky behavior, rather than a direct cause of unprotected sex [28].

The results among community males suggest that those who used substances were more cautious than were those who used no substances during their last sexual encounter. This was somewhat unexpected. However, previous research does report negative associations between substance use and protected sex. In a study to test event-level associations between substance use and condom use among homeless and runaway youth, results showed that those who used crack were more likely than those who used no substances to use a condom [29]. It is not clear why those who used substances would be less likely to have unprotected sex. One explanation for this could be related to the type of sexual partner. The literature reports a higher likelihood of condom use with casual partners. It is possible that community males in this study used condoms despite using AOD because of the nature of the relationship with their sexual partners. This notion is supported by the fact that males often have more power than their female counterparts and consequently are the decision makers with regards to condom use [30]. In addition, there may be some contextual variables that we did not examine, which may play a role in the AOD-condom use relationship during a sexual encounter. Further research is needed to explain counter intuitive findings such as ours.

Several limitations of the present study deserve mention. First, this study was conducted among adolescents and young adults in Colorado, most whom were white. Therefore, caution should be exercised when generalizing these results to diverse populations. Second, participants were only asked if they used substances the last time they had unprotected and protected sex, but they were not asked how much they consumed. Variations in the pharmacological effects of different substances might affect how much of them are used in a sexual situation, as well as their effects on condom use [31]. A third limitation is that although the case-crossover design is meant to remove the need to control for characteristics that are not timevarying, including ethnicity, socioeconomic status, impulsivity, etc.), it is possible that these variables differed across the groups (male/ female, community/clinical) and might they might explain differences between groups. Fourthly, the two events could differ in several other contextual ways, such as the partner type involved (therefore condom use), partner characteristics, and the context of the sexual event. Thus, there are some time-varying covariates that are not controlled for in the analyses. Lastly, the small size of the clinical sample could have limited the likelihood of a relationship being detected, given the low power. Despite these limitations, this study adds to the literature on event-level relationship between substance use and sexual risk behavior, specifically by including a clinical sample, stratifying by gender, and having a larger sample than most event-level studies on substance use and unprotected sex.

The findings from this study do not support the assumption that use of AOD during a sexual encounter leads to risky sexual behavior, and they therefore have important implications for public health. These findings caution STI/HIV preventive programs that largely focus on the consequences of engaging in sex while under the influence of AOD. The link between substance use and sexual behavior is multifaceted and encompasses several other important factors, including the type and amount of substances, components of personality, context of sexual encounter and type of relationship. Preventive programs need to take such factors into consideration as they design educational messages.

\section{References}

1. Centers for Disease Control and Prevention. Sexually Transmitted Disease Surveillance 2014. Atlanta: U.S. Department of Health and Human Services. 2015.

2. Centers for Disease Control and Prevention. HIV Prevalence EstimatesUnited States, 2006. MMWR Morb Mortal Wkly Rep. 2008; 57: 1073-1076.

3. Ward H, Rönn M. The Contribution of STIs to the Sexual Transmission of HIV. Curr Opin HIV AIDS. 2010; 54: 305-310.

4. Howard J, Taylor JA, Ganikos ML, Holder HD, Godwin DF, Taylor ED. An Overview of Prevention Research: Issues, Answers, and New Agendas. Public Health Rep. 1988; 103: 674-683.

5. Ostrow DG, Beltran E, Joseph G, DiFranceisco W, Wesch J, Chmiel JS. Recreational Drugs and Sexual Behavior in the Chicago MACS/CCS Cohort of Homosexually Active Men. Chicago Multicenter AIDS Cohort Study (MACS)/Coping and Change Study. J Subst Abuse. 1993; 5: 311-325.

6. Purcell DW, Parsons JT, Halkitis PN, Mizuno Y, Woods WJ. Substance Use and Sexual Transmission Risk Behavior of HIV-Positive Men Who Save Sex with Men. J Subst Abuse. 2001; 13: 185-200.

7. Kaiser Family Foundation. Substance Use and Risky Sexual Behavior: Attitudes and Practices among Adolescents and Young Adults. 2000.

8. Leigh BC, Stall R. Substance Use and Risky Sexual Behavior for Exposure to HIV. Issues in Methodology, Interpretation, and Prevention. Am. Psychol. 1993; 48: 1035-1045.

9. Morrison DM, Gillmore MR, Hoppe MJ, Gaylord J, Leigh BC, Rainey D Adolescent Drinking and Sex: Findings from a Daily Diary Study. Perspect Sex Reprod Health. 2003; 35: 162-168.

10. Scott-Sheldon LAJ, Carey MP, Vanable PA, Senn TE, Coury-Doniger P. Urban MA. Alcohol Consumption, Drug Use, and Condom Use among STD Clinic Patients. J Stud Alcohol Drugs. 2009; 70: 762-770.

11. Bryan AD, Schmiege SJ, Magnan RE. Marijuana Use and Risky Sexual Behavior among High-Risk Adolescents: Trajectories, Risk Factors, and Event-Level Relationships. Dev. Psychol. 2012; 48: 1429-1442.

12. Substance Abuse and Mental Health Services Administration, Results from the 2013 National Survey on Drug Use and Health: Summary of National Findings, NSDUH Series H-48, HH Publication No. (SMA) 14-4863. Rockville, MD: Substance Abuse and Mental Health Services Administration. 2014.

13. Bontempi JB, Mugno R, Bulmer SM, Danvers K, Vancour ML. Exploring Gender Differences in the Relationship Between HIVISTD Testing and Condom Use Among Undergraduate College Students. J Health Educ. 2009; 40: $97-105$

14. Calsyn DA, Peavy M, Wells EA, Campbell AN, Hatch-Maillette MA, Greenfield SF et al. Differences between Men and Women in Condom Use, Attitudes, and Skills in Substance Abuse Treatment Seekers. Am J Addict. 2013; 2: 150-157.

15. Scheidt DM, Windle M. The Alcoholics in Treatment HIV Risk (ATRISK) Study: Gender, Ethnic and Geographic Group Comparisons. J. Stud. Alcohol. 1995; 56: 300-308

16. Lansky A, Finlayson T, Johnson C, Holtzman D, Wejnert C, Mitsch A, et al. Estimating the Number of Persons Who Inject Drugs in the United States by Meta-Analysis to Calculate National Rates of HIV and Hepatitis C Virus Infections. PLoS ONE. 2014; 9: 97596.

17. Kahn JA, Kaplowitz RA, Goodman E, Emans SJ. The Association between Impulsiveness and Sexual Risk Behaviors in Adolescent and Young Adult Women. J. Adolesc. Health.2002; 30: 229-232. 
18. Rhea SA, Gross AA, Haberstick BC, Corley RP. Colorado Twin Registry. Twin Res. Hum. Genet. 2006; 9: 941-949.

19. Petrill SA, Rhea SA. Memory Ability during Middle Childhood and Early Adolescence in the Colorado Adoption Project. Petrill SA, Plomin R, DeFries JC, Hewitt JK, editors. In: Nature, Nurture, and the Transition to Adolescence. Oxford: Oxford University Press. 2003; 166-184.

20. Stallings MC, Corley RP, Hewitt JK, Krauter KS, Lessem JM, Mikulich SK et al. A genome-wide search for quantitative trait loci influencing substance dependence vulnerability in adolescence. Drug Alcohol Depend. 2003; 70 295-307.

21. Booth RE, Mikulich-Gilbertson SK, Brewster JT, Salomonsen-Sautel S Semerik O. Predictors of self-reported HIV Infection among Drug Injectors in Ukraine. J Acquir Immune Defic Syndr. 2004; 35: 82-88.

22. R Development Core Team. R: A Language and Environment for Statistical Computing. Vienna, Austria: The R Foundation for Statistical Computing 2011.

23. Matser M, Heiligenberg R, Geskus T, Heijman N, Low M, Kretzschmar M, et al. The Importance of Partnership Factors and Individual Factors Associated with Absent or Inconsistent Condom Use in Heterosexuals: A Cross-Sectiona Study. Sex. Transm. Infect. 2014; 90: 325-331.

24. Charania MR, Crepaz N, Guenther-Gray C, Henny K, Liau A., Willis LA, et al. Efficacy of Structural-Level Condom Distribution Interventions: A MetaAnalysis of US and International Studies, 1998-2007. AIDS Behav. 2011; 15: $1283-1297$
25. Fortenberry JD, Orr DP, Katz BP, Brizendine EJ, Blythe MJ. Sex under the Influence. A Diary Self-Report Study of Substance Use and Sexual Behavior Among Adolescent Women. Sex Transm Dis. 1997; 24: 313-319.

26. Hittner JB, Swickert R. Sensation Seeking and Alcohol Use: A Meta-Analytic Review. Addict Behav. 2006; 31: 1383-1401.

27. Kahn JA, Kaplowitz RA, Goodman E, Emans SJ. The Association between Impulsiveness and Sexual Risk Behaviors in Adolescent and Young Adult Women. J. Adolesc. Health. 2002; 30: 229-232.

28. Muchimba M, Haberstick BC, Corley RP, McQueen MB. Frequency of Alcohol Use in Adolescence as a Marker for Subsequent Sexual Risk Behavior in Adulthood. J. Adolesc. Health. 2013; 53: 215-221.

29. Bailey SL, Camlin CS, Ennett ST. Substance Use and Risky Sexual Behavior among Homeless and Runaway Youth. J. Adolesc. Health. 1998; 23: 378388.

30. Harvey SM, Bird ST. What Makes Women Feel Powerful? An Exploratory Study of Relationship Power and Sexual Decision-Making with AfricanAmerican at Risk For HIVISTIs. Women Health. 2004; 39: 1-18.

31. Leigh BC, Ames SL, Stacy AW. Alcohol, Drugs, and Condom Use among Drug Offenders: An Event-Based Analysis. Drug Alcohol Depend. 2008: 93: 38-42.
Austin J HIV/AIDS Res - Volume 4 Issue 1 - 2017

ISSN : 2380-0755 | www.austinpublishing group.com

Muchimba et al. @ All rights are reserved
Citation: Muchimba M, Burton M, Haberstick BC, Corley RP, Hopfer C and McQueen M. Event-Level Substance Use and Unprotected Sex among Young Adults: A Case-Crossover Analysis. Austin J HIV/AIDS Res. 2017; 4(1): 1034. 